\title{
Propuesta de adaptación del reglamento de fútgolf para personas con parálisis cerebral*
}

\author{
[Artículos]
}

Víctor Hernández-Beltrán**

José M. Gamonales ${ }^{* * *}$

Luisa Gámez-Calvo*****

Jesús Muñoz-Jiménez ${ }^{* * * * *}$

Recibido 15 de enero de 2021

Aceptado: o5 de abril de 2021

Citar como:

Hernández-Beltrán, V., Gamonales, J.M., Gámez-Calvo, L. y Muñoz-Jiménez, J. (2021). Propuesta de adaptación del reglamento de fútgolf para personas con parálisis cerebral. Revista de Investigación Cuerpo, Cultura y Movimiento, 11(2). https://doi.org/10.15332/2422474X.6759

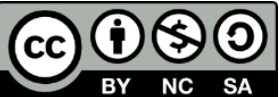

\footnotetext{
* Avances de investigación. Este trabajo ha sido parcialmente subvencionado por la Ayuda a los Grupos de Investigación (GR18170), de la Junta de Extremadura (Consejería de Empleo e Infraestructuras), con la aportación de la Unión Europea a través de los Fondos Europeos de Desarrollo Regional (FEDER). Grupo de Optimización del Entrenamiento y Rendimiento Deportivo (GOERD) de la Facultad de Ciencias del Deporte, Universidad de Extremadura. Cáceres, España.

** Graduado en Educación Primaria, doctorando en Ciencias del Deporte, Universidad de Extremadura, España. Correo electrónico: vhernandpw@alumnos.unex.es; ORCID: https://orcid.org/0000-0002-7449-5734

${ }^{* * *}$ Doctor en Ciencias del Deporte, profesor en la Facultad de Educación, Universidad de Extremadura, España. Correo electrónico: josemartingamonales@gmail.com; ORCID: https://orcid.org/0000-0002-2444-1535

${ }_{* * * *}$ Graduado en Ciencias de la Actividad Física y el Deporte, doctorando en Ciencias del Deporte, Universidad de Extremadura, España. Correo electrónico: Igamezna@alumnos.unex.es; ORCID: https://orcid.org/0000-0002-5205-8349

${ }^{* * * * *}$ Doctor en Ciencias del Deporte, profesor en la Facultad de Ciencias del Deporte, Universidad de Extremadura, España. Correo electrónico: suliwan@unex.es; ORCID: https://orcid.org/00000003-1283-5227
}

Revista de Investigación Cuerpo, Cultura y Movimiento

ISSN: 2248-4418 | e-ISSN: 2422-474X | DOI: https://doi.org/10.15332/2422474X

Vol. 11 N.0 2 | julio-diciembre de 2021 


\section{Resumen}

El fútgolf no se encuentra adaptado entre las modalidades deportivas de la Federación Española de Deportes de Personas con Parálisis Cerebral y Daño Cerebral Adquirido. Por tanto, el objetivo del presente trabajo es llevar a cabo una propuesta de adaptación del reglamento de fútgolf para las personas con parálisis cerebral en función de la clasificación funcional de los jugadores de fútbol. Tomando como referencia el reglamento de fútgolf convencional, se ha realizado una adaptación de materiales, instalaciones deportivas y aspectos técnico-tácticos. La práctica del deporte por parte de las personas con discapacidad en general y con parálisis cerebral en particular puede tener diferentes beneficios en la condición física, así como a nivel psicológico, físico, social, laboral, funcional y recreativo que mejoran la calidad de vida y facilitan la inclusión de esta población.

Palabras clave: fútbol, discapacidad, normativa, inclusión.

\section{Proposal to adapt footgolf rules for people with cerebral palsy}

\section{Abstract}

Footgolf is not adapted among the sports modalities of the Spanish Sports Federation for People with Cerebral Palsy and Acquired Brain Damage. Therefore, the objective of this work is to carry out a proposal to adapt footgolf rules for people with cerebral palsy based on the functional classification of soccer players. Taking as reference the rules of conventional footgolf, an adaptation of materials, sports facilities, and technical-tactical aspects has been made. The practice of the sport by people with disabilities in general and with cerebral palsy in particular can have different benefits in the physical condition, as well as at psychological, physical, social, occupational, functional and recreational 
level, that improve the quality of life and facilitate the inclusion of this population.

Keywords: soccer, disability, regulations, inclusion.

\section{Introducción}

En los últimos años, la práctica físico-deportiva para personas con discapacidad está siendo reconocida socialmente (Pérez-Tejero et ál., 2012) e incluso existe una creciente tendencia en relación con la cantidad de modalidades deportivas y desarrollo de eventos deportivos (Benítez et ál., 2012; Shapiro et ál., 2012). Por ello, la actividad física deportiva puede dar respuesta a diferentes necesidades: terapéuticas, educativas, recreativas y competitivas (Sanz-Rivas y Reina-Vaíllo, 2012), y puede considerarse como una práctica adaptada al colectivo de personas que la realizan, basada en la realización de una serie de modificaciones de la estructura del deporte para permitir su práctica por parte de deportistas con discapacidad (Gamonales, 2016; Gamonales, 2020; Muñoz-Jiménez et ál., 2018). Además, mediante la práctica de deporte adaptado se favorece una formación integral, lo que permite el desarrollo de diferentes conceptos relacionados con la personalidad, reduce los niveles de ansiedad y estrés, y mejora el desarrollo de su bienestar personal (GutiérrezSanmartín, 2004). Por ello, para facilitar la práctica de una modalidad deportiva determinada a las personas con discapacidad, es necesario llevar a cabo un proceso de adaptación de esta (Gamonales, 2017; Reina, 2010). En relación con la adaptación del reglamento, el baloncesto en silla de ruedas es uno de los deportes que ha adaptado su normativa para poder ser practicado por personas con discapacidad física, con el objetivo de dar cabida a la incorporación de los desplazamientos en silla de ruedas durante el juego (De Sousa-Peña et ál., 2020). En otros deportes, como en el fútbol, se han llevado a cabo adaptaciones de sus reglas enfocadas a 
facilitar su práctica por parte del colectivo con discapacidad visual (Gamonales, Muñoz-Jiménez et ál., 2018) y para personas con parálisis cerebral o daño cerebral adquirido (Gamonales, 2020; Gamonales, León et ál., 2019). También se han propuesto adaptaciones de deportes para deportistas con discapacidad intelectual, como por ejemplo el balonmano (Gamonales, Hidalgo-Murillo et ál., 2018).

Por otro lado, existen deportes que se han creado específicamente para ser practicados por personas que presentan algún tipo de discapacidad (Moya, 2014). Como ejemplo, encontramos la Boccia, deporte para personas con parálisis cerebral o daño cerebral adquirido (PC) (Lapresa et ál., 2017), o el goalball, modalidad deportiva creada especialmente para las personas con discapacidad visual (Federación Española de Deportes ara Ciegos [FEDC], 2020). Tanto los deportes específicos como adaptados para personas con discapacidad se están introduciendo en las aulas de Educación Física como parte de una formación inclusiva (Gamonales, 2017). Sin embargo, no todos los deportes convencionales han sido adaptados para personas con discapacidad, en general, o para personas con PC, en particular.

La PC se caracteriza por una pérdida del control muscular debido a un desorden producido en el desarrollo del cerebro durante la formación del feto (Richards y Malouin, 2013). Las deficiencias relacionadas con la PC pueden ser muy dispares (Hernández-Vázquez et ál., 2016) y afectan la comunicación, la conducta y, en algunos casos, a la percepción cognitiva (Bax et ál., 2005). Por tanto, dependiendo de la afección de la PC y del grado de movimiento que puede desarrollar la persona, en el fútbol se establecen varias categorías funcionales $(\mathrm{CF})$ para las personas con $\mathrm{PC}$ (tabla 1). El grado de afección es mayor en $\mathrm{CF}_{1}$ y menor en $\mathrm{CF}_{3}$, por lo que la posibilidad de movimiento irá aumentando de un nivel de clasificación al siguiente. 
Tabla 1. Clasificación funcional en fútbol para personas con PC

\begin{tabular}{|c|c|c|}
\hline Clase funcional & Descripción & Movilidad \\
\hline CF1 & $\begin{array}{c}\text { Diplejía, doble } \\
\text { hemiplejia y distonia }\end{array}$ & $\begin{array}{l}\text { - Tienen dificultad a la hora de realizar giros. } \\
\text { - Pueden correr distancias cortas. } \\
\text { - La longitud de los pasos está afectada. }\end{array}$ \\
\hline CF2 & $\begin{array}{l}\text { Ataxia, parálisis } \\
\text { cerebral mixta y } \\
\text { hemiplejia }\end{array}$ & $\begin{array}{l}\text { - Dificultad a la hora de cambiar de dirección } \\
\text { - El equilibrio está afectado debido a la parálisis de los } \\
\text { miembros de un lado del cuerpo } \\
\text { - Presentan cojera o asimetría en la longitud de los } \\
\text { pasos, lo que afecta los golpeos en situaciones difíciles. }\end{array}$ \\
\hline CF3 & $\begin{array}{l}\text { Diplejía asimétrica, } \\
\text { hemiplejia de grado } \\
1 \text { o 2, monoplejía } \\
\text { con un grado grande } \\
\text { de espasticidad }\end{array}$ & $\begin{array}{l}\text { - La acción de correr no está afectada. } \\
\text { - Debido a la espasticidad de los miembros, pueden } \\
\text { presentar una ligera cojera, pero sin llegar a afectar al } \\
\text { paso. } \\
\text { - Golpes cortos pueden verse afectados en la dirección } \\
\text { en los sujetos con ataxia. }\end{array}$ \\
\hline
\end{tabular}

Fuente: elaboración propia.

El programa deportivo de la Federación Española de Deportes de Personas con Parálisis Cerebral y Daño Cerebral Adquirido (FEDPC) contiene los siguientes deportes: atletismo, boccia, deportes de invierno (esquí alpino y esquí nórdico), fútbol, natación y slalom en silla de ruedas. Este último es uno de los deportes mejor adaptados para personas con mayor grado de afectación funcional (FEDPC, 2020).

Como consecuencia del proceso de integración a nivel internacional de los deportes adaptados, existen federaciones unideportivas que cuentan con modalidades destinadas a las personas con discapacidad, en concreto: bádminton, remo, triatlón, ciclismo, hípica, piragüismo, taekwondo, tenis, tenis de mesa, tiro con arco y vela. Entre todas estas modalidades deportivas que han adaptado su normativa a las personas con PC, no se encuentra el fútgolf. Este deporte surgió en Holanda en el año 2009, con la finalidad de facilitar la rehabilitación y socialización de aquellas personas con discapacidad y que buscan una participación más activa dentro de nuestra sociedad (Reis et ál., 2020). 
Hoy en día, son 36 los países que están inscritos en la Federación Internacional de Fútgolf (FIFG) y que tienen equipos participantes en las diferentes competiciones que se desarrollan alrededor del mundo. Esta federación es la responsable de organizar los campeonatos del mundo desde su origen. Además, de manera independiente, se desarrollan torneos a nivel continental en América, Asia o Europa, como el campeonato de Europa de Fútgolf, organizado en París en 2017 (FIFG, 2019). Adaptar este deporte a cualquier colectivo de personas, independientemente de su edad, género o condición, así como para personas con discapacidad, puede aumentar el número de practicantes. Por tanto, en el presente trabajo se va a desarrollar una propuesta de adaptación de fútgolf para personas con PC o con daño cerebral adquirido (FútgolfPC), tomando como referencia la clasificación funcional del fútbol a 7, adaptado a este colectivo (Fa7PC). En la tabla 2, se recogen algunas de las normas básicas del deporte establecidas por la FIFG (2019). 
Tabla 2. Principales reglas del fútgolf

\begin{tabular}{|c|c|c|c|}
\hline \multicolumn{2}{|c|}{ Aspecto } & \multicolumn{2}{|r|}{ Descripción } \\
\hline \multicolumn{2}{|c|}{ Objetivo de juego } & \multicolumn{2}{|c|}{$\begin{array}{l}\text { Llevar la bola con el menor número de golpes posible, desde el punto de salida hasta la zona verde donde se } \\
\text { encuentra el hoyo. Los jugadores deben evitar los obstáculos que se encuentran en la pista, como agua o } \\
\text { agujeros, para lograr llegar a la zona verde. }\end{array}$} \\
\hline \multicolumn{2}{|c|}{ Árbitros } & \multicolumn{2}{|r|}{ No existe supervisión de árbitros. } \\
\hline \multirow{2}{*}{\multicolumn{2}{|c|}{ Tipos de juego }} & \multirow{2}{*}{\multicolumn{2}{|c|}{$\begin{array}{l}\begin{array}{l}\text { Stroke play. Todos los jugadores juegan contra todos, y el que complete la sección en los menos golpes es el } \\
\text { ganador. }\end{array} \\
\text { Match play. Se juega en equipo, es el equipo que menos golpeos utilice para completar la sección el ganador. }\end{array}$}} \\
\hline & & & \\
\hline \multicolumn{2}{|c|}{ Penalizaciones } & \multicolumn{2}{|c|}{$\begin{array}{c}\text { El incumplimiento de las normas de juego conllevará la suma de un golpeo al número que acumule ese } \\
\text { momento el jugador. }\end{array}$} \\
\hline \multirow{5}{*}{ Obstrucciones } & $\begin{array}{l}\text { Obstrucciones } \\
\text { sueltas }\end{array}$ & \multicolumn{2}{|c|}{$\begin{array}{l}\text { Cualquier impedimento suelto puede ser movido sin ser penalizado, excepto si el obstáculo está en contacto } \\
\text { con la pelota. }\end{array}$} \\
\hline & $\begin{array}{l}\text { Obstrucciones } \\
\text { movibles }\end{array}$ & \multicolumn{2}{|c|}{$\begin{array}{l}\text { Si el balón no está dentro del obstáculo, este puede ser movido. } \\
\text { Si la pelota descansa sobre el obstáculo, se procede a levantar la pelota y mover el obstáculo. } \\
\text { Ambas acciones no acarrean penalización. }\end{array}$} \\
\hline & \multirow{3}{*}{$\begin{array}{l}\text { Obstrucciones } \\
\text { inamovibles }\end{array}$} & Interferencia & Cuando la pelota está dentro o sobre el obstáculo. \\
\hline & & \multirow[t]{2}{*}{ Alivio } & $\begin{array}{l}\text { Si el balón está sobre el suelo, el jugador podrá levantar la pelota y moverse dos pasos } \\
\text { (nunca en la dirección de hoyo). }\end{array}$ \\
\hline & & & Si la pelota se encuentra en un búnker, debe ser golpeada dentro del mismo. \\
\hline \multirow{3}{*}{\multicolumn{2}{|c|}{$\begin{array}{l}\text { Golpeo (según } \\
\text { localización de la bola) }\end{array}$}} & $\begin{array}{l}\text { Terreno en } \\
\text { reparación }\end{array}$ & $\begin{array}{l}\text { Regla de la perpendicularidad. Para ello, se establecerá una línea imaginaria entre el punto } \\
\text { de la pelota y el hoyo con la finalidad que se extienda una línea (línea imaginaria) hacia } \\
\text { ambos costados del terreno de juego. }\end{array}$ \\
\hline & & $\begin{array}{l}\text { Obstáculo de } \\
\text { agua frontal }\end{array}$ & Con penalización, el jugador deberá sacar la pelota del agua y retrocederá dos metros. \\
\hline & & $\begin{array}{l}\text { Obstáculo de } \\
\text { agua lateral }\end{array}$ & Se golpeará la pelota desde la zona donde se encuentre, con un golpe de penalización. \\
\hline
\end{tabular}

Revista de Investigación Cuerpo, Cultura y Movimiento

ISSN: 2248-4418 | e-ISSN: 2422-474X | DOI: https://doi.org/10.15332/2422474X

Vol. 11 N.0 2 | julio-diciembre de 2021 


\section{Aspecto}

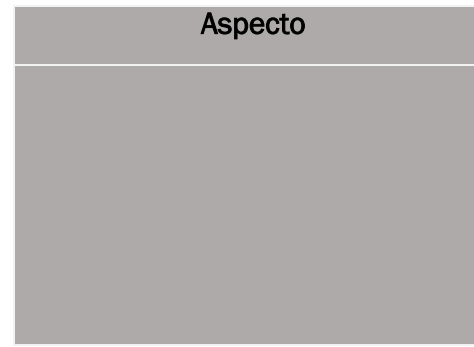

Fuente: elaboración propia.

\section{Descripción}

Si la pelota no cae en treinta segundos, se aplica la regla de la perpendicularidad mientras el árbol está dentro de los límites. Si está fuera del terreno de juego, se realiza el golpe de nuevo con penalización.

En altura

En bunker

Flotando
Se debe golpear la pelota desde el interior del búnker sin carrera previa.

El jugador decide si golpearla. Si la pelota se liberará del obstáculo, el jugador será penalizado. 


\section{Método}

Las modalidades deportivas adaptadas a las personas con discapacidad están destinadas a sujetos que presentan algún déficit motor, sensorial o cognitivo (Germán, 2001), con el fin de llevar una vida activa, siendo un elemento positivo para construir una sociedad accesible (Gamonales, 2017; Muñoz-Jiménez et ál., 2018). El fútgolf tiene como finalidad conseguir llevar la pelota desde el punto de inicio hasta el hoyo final en el menor número de golpes (FIFG, 2019). Por lo tanto, la adaptación del reglamento de fútgolfPC deberá tener en cuenta aspectos fundamentales, como balón, equipo, modelo de juego, reglamento, tiempos y acciones técnicas-tácticas, al igual que sucede en la adaptación realizada en balonmano para jugadores con discapacidad intelectual (Gamonales, Hidalgo-Murillo et ál., 2018).

Por ello, la práctica del fútgolfPC puede presentar diferentes beneficios tanto a nivel cognitivo, como a nivel social, además de mejorar la condición física. Favorece la inclusión en el deporte, entendiendo el término inclusión como la posibilidad de que todos los participen de manera plena y activa, y desarrollen las actividades todos juntos en los mismos espacios independientemente de las capacidades personales (Gamonales, 2016; Montilla-Reina, 2019); es una oportunidad de derribar prejuicios aun presentes en la sociedad. Puede ayudar a fortalecer la afectividad, la emotividad, la motivación o la autosuperación de los jugadores (Germán, 2001), puesto que los deportistas deberán emplear estrategias e inteligencia para conseguir el éxito.

Por último, el desarrollo de esta propuesta parte del análisis de la oferta deportiva actual para las personas con PC. Posteriormente, se realiza una clasificación funcional similar a la utilizada en Fa7 para personas con PC. Por último, teniendo en cuenta el reglamento de la disciplina y las 
características del colectivo, se lleva a cabo una adaptación en el reglamento del deporte, que se centra en los aspectos técnico-tácticos, el material y las instalaciones deportivas, siempre considerando la clasificación funcional propuesta. De esta manera, se está favoreciendo el desarrollo de la disciplina para ser practicada por personas con o sin discapacidad.

\section{Resultados y discusión}

El fútgolf es una modalidad deportiva novedosa y de reciente creación que no ha sido adaptada para las personas con PC, por lo que teniendo en cuenta las diferentes clasificaciones funcionales establecidas anteriormente y la normativa de la FIFG, se ha propuesto una adaptación del reglamento para este colectivo (tabla 3), teniendo en cuenta los aspectos a modificar sobre lo establecido por Gamonales (2017) y MuñozJiménez et ál. (2018).

Tabla 3. Adaptaciones del reglamento de fútgolfPC

\begin{tabular}{|c|c|c|c|c|}
\hline \multirow{2}{*}{\multicolumn{2}{|c|}{ Aspecto }} & \multicolumn{3}{|c|}{ Descripción } \\
\hline & & CF1 & CF2 & CF3 \\
\hline \multicolumn{2}{|c|}{ Material } & $\begin{array}{l}\text { El balón será de una } \\
\text { dureza menor para } \\
\text { facilitar el golpeo. El } \\
\text { resto de material } \\
\text { será propio de cada } \\
\text { jugador. }\end{array}$ & $\begin{array}{l}\text { El balón será de una } \\
\text { dureza menor para } \\
\text { facilitar el golpeo. El } \\
\text { resto de material } \\
\text { será propio de cada } \\
\text { jugador. }\end{array}$ & $\begin{array}{l}\text { No habrá } \\
\text { adaptaciones en } \\
\text { respecto a la pelota. } \\
\text { El resto de material } \\
\text { será propio de cada } \\
\text { jugador. }\end{array}$ \\
\hline \multirow{3}{*}{$\begin{array}{l}\text { Aspectos } \\
\text { técnico- } \\
\text { táctico }\end{array}$} & Objetivo & \multicolumn{3}{|c|}{$\begin{array}{c}\text { El objetivo del juego será el mismo: conseguir meter la bola en la } \\
\text { zona verde en el mínimo de golpes posibles. }\end{array}$} \\
\hline & Árbitro & \multicolumn{3}{|c|}{$\begin{array}{l}\text { El juego se desarrollará sin la presencia de árbitros. En el campo de } \\
\text { juego podrán estar los entrenadores con los jugadores garantizando } \\
\text { el juego limpio y responsable. Las decisiones del juego la tomarán } \\
\text { los jugadores en la medida lo posible, en el caso de que no sea } \\
\text { posible serán los entrenadores los que lleguen a un acuerdo. }\end{array}$} \\
\hline & Tipo de juego & $\begin{array}{l}\text { Mismo tipo de juego } \\
\text { sin adaptaciones. Se } \\
\text { permitirán relevos } \\
\text { en Match play. para } \\
\text { evitar en cansancio } \\
\text { de los jugadores. }\end{array}$ & $\begin{array}{l}\text { No se llevarán a } \\
\text { cabo adaptaciones } \\
\text { en los tipos de } \\
\text { juego en CF2. }\end{array}$ & $\begin{array}{c}\text { No se llevarán a } \\
\text { cabo adaptaciones } \\
\text { en los tipos de juego } \\
\text { en CF3. }\end{array}$ \\
\hline
\end{tabular}




\begin{tabular}{|c|c|c|c|}
\hline \multirow{2}{*}{ Aspecto } & \multicolumn{3}{|c|}{ Descripción } \\
\hline & CF1 & CF2 & CF3 \\
\hline Penalizaciones & \multicolumn{3}{|c|}{$\begin{array}{c}\text { No habrá adaptaciones en relación con las penalizaciones en } \\
\text { ninguna de las CF. En el caso de las obstrucciones, se seguirán las } \\
\text { adaptaciones realizadas en las mismas. }\end{array}$} \\
\hline Obstrucciones & $\begin{array}{c}\text { Todos los obstáculos } \\
\text { pueden ser retirados } \\
\text { para facilitar el } \\
\text { golpeo sin obtener } \\
\text { penalización, } \\
\text { siempre y cuando la } \\
\text { pelota no toque el } \\
\text { obstáculo. Si la } \\
\text { pelota se encuentra } \\
\text { sobre el obstáculo } \\
\text { está será alejada del } \\
\text { mismo (en dirección } \\
\text { contraria al hoyo) } \\
\text { para facilitar el } \\
\text { golpeo de nuevo. }\end{array}$ & $\begin{array}{l}\text { Si la pelota está } \\
\text { sobre un obstáculo, } \\
\text { pueden golpearla si } \\
\text { es posible con la } \\
\text { ayuda del } \\
\text { entrenador sin } \\
\text { recibir penalización. } \\
\text { En el caso de que } \\
\text { haya que retirar el } \\
\text { obstáculo o } \\
\text { recolocar la pelota, } \\
\text { el jugador recibirá } \\
\text { un golpeo de } \\
\text { penalización. }\end{array}$ & $\begin{array}{l}\text { No se realizarán } \\
\text { adaptaciones. Se } \\
\text { siguen las mismas } \\
\text { reglas que en el } \\
\text { fútgolf. }\end{array}$ \\
\hline Tipo de golpeo & \multicolumn{3}{|c|}{$\begin{array}{c}\text { La pelota será liberada del obstáculo en dirección contraria al hoyo } \\
\text { o siguiendo la norma de la perpendicularidad. }\end{array}$} \\
\hline $\begin{array}{l}\text { Instalaciones } \\
\text { deportivas }\end{array}$ & \multicolumn{3}{|c|}{$\begin{array}{l}\text { No se realizarán adaptaciones en el terreno de juego en ninguna de } \\
\text { las CF. Si algún jugador presenta dificultad a la hora de desplazarse } \\
\text { por la pista, podrá utilizar silla de ruedas. El hoyo final tendrá un } \\
\text { tamaño mayor al establecido ( } 52 \text { centímetros de diámetro y } 35 \\
\text { centímetros de profundidad). Se mantendrá la misma profundidad, } \\
\text { pero el diámetro del hoyo se aumentará a } 1 \text { metro. }\end{array}$} \\
\hline
\end{tabular}

CF1: clasificación funcional 1; CF2: clasificación funcional 2; CF3: clasificación funcional 3.

Fuente: elaboración propia.

En la literatura científica, los documentos relacionados con el fútgolf son escasos (Reis et ál., 2020). Es un deporte practicado en numerosos países a nivel mundial, pero aún no consta de una adaptación para las personas con PC, por lo que es novedoso adaptar el deporte con el fin de que este colectivo tenga una oferta deportiva más amplia. Para ello, se ha realizado una adaptación del reglamento del fútgolfPC teniendo en cuenta la clasificación funcional de cada uno de los jugadores, con el objetivo de que en un futuro la FEDPC pueda contar con un reglamento propio que le permita incluir este deporte en su catálogo de modalidades. Con el presente trabajo, además, se pretende dar a conocer a los profesionales e 
investigadores de habla hispana las principales modificaciones y estrategias que se han llevado en dicha disciplina para ser practicada por personas con PC.

Según Muñoz-Díaz (2009), la actividad física tiene muchos beneficios en las personas con PC: sociales, afectivos, competitivos, motores, cognitivos y de autonomía personal. Como la PC afecta al sistema musculoesquelético, los objetivos de la actividad física deben ir enfocados a tonificar la musculatura, adquirir nuevos y correctos patrones motores (caminar de la manera más estable posible), buscar un óptimo control postural o mejorar la propiocepción y coordinación (Benítez et ál., 2012). Por otro lado, se debe favorecer y ampliar la oferta deportiva a las personas con discapacidad que aporte a la plena inclusión en la sociedad, al igual que evite en la medida de los posible el sedentarismo en las personas con PC. Hay que tener en cuenta que, en el caso de las personas con discapacidad, existe una vinculación directa entre la práctica físicodeportiva y la mejora de la calidad de vida, con la finalidad de mejorar la inclusión social (Shalock y Verdugo, 2003), la condición física y promover el bienestar psicológico (Murphy y Carbone, 2008). Por ello, el fútgolfPC será una excelente herramienta para trabajar lograr este objetivo, en un entorno de colaboración y confianza en los demás, con base en el compañerismo y la solidaridad.

Respecto a las adaptaciones del fútgolfPC, se ha tenido en cuenta las características de cada grupo funcional, llevando a cabo una serie de adaptaciones tanto en el reglamento como en los aspectos técnico-tácticos del deporte, con lo cual se permite su práctica, como sucede en la propuesta de adaptación del reglamento de balonmano para jugadores con discapacidad intelectual (Gamonales, Hidalgo-Murillo et ál., 2018), o en fútbol para personas con discapacidad visual (Gamonales, 2020; Gamonales, Muñoz-Jiménez et ál., 2018). 
Para llevar a cabo las adaptaciones para la práctica del fútgolfPC, se ha tenido en cuenta los aspectos por adaptar de un deporte convencional establecidos por Gamonales (2017): reglamento, aspecto técnico-tácticos, material e instalaciones deportivas. Estas adaptaciones están en función de la clasificación funcional utilizada en Fa7PC, con la finalidad de asegurar la igualdad en la participación y evitar situaciones de abandono deportivo de los deportistas con discapacidad por no poder desarrollar competiciones equilibradas desde el punto de vista del rendimiento funcional. Existen estudios que demuestran que las personas con discapacidad abandonan la práctica físico-deportiva por motivos de carácter intrapersonal, falta de ofertas de actividades (falta de motivación por la falta de nuevas propuestas) y económicos (Úbeda-Colomer et ál., 2018).

Por último, sería importante llevar a cabo más propuestas de adaptación de reglamentos de otras modalidades deportivas para favorecer la inclusión de las personas con discapacidad. En cuanto a las limitaciones de este estudio, la propuesta de reglamento no se ha llevado a cabo aún, pero se encuentra fundamentada en la literatura científica y demuestra los múltiples beneficios del deporte adaptado en las personas con discapacidad. Además, se ha utilizado una clasificación funcional predeterminada para una modalidad deportiva con gran repercusión para las personas con PC como es el fútbol a 7.

\section{Conclusiones}

La propuesta de reglamento para el fútgolf adaptado a personas con PC tiene como objetivo fomentar la inclusión de este colectivo en el deporte y en la sociedad, y evitar el sedentarismo de las personas con PC. Además, permitirá incrementar los niveles de autoestima de las personas con discapacidad. 
La adaptación del reglamento permite la práctica del fútgolfPC, al fomentar la participación de este colectivo en el ámbito deportivo y mejorar la actitud del resto de la sociedad, ya que también se podría practicar de manera inclusiva por personas con diferentes capacidades.

Para aumentar la práctica físico-deportiva por parte de las personas con discapacidad es importante que las federaciones realicen modificaciones en los reglamentos de aquellas modalidades deportivas que no hayan sido adaptadas.

Por último, será necesario desarrollar estudios específicos relacionados con el fútgolfPC, con la finalidad de conocer la idoneidad de la modalidad deportiva para personas con parálisis o daño cerebrales adquirido.

\section{Referencias}

Bax, M., Goldstein, M., Rosenbaum, P., Leviton, A., Paneth, N., Dan, B., Jacobsson, B. y Damiano, D. (2005). Proposed definition and classification of cerebral palsy. Developmental Medicine and Child Neurology, 47(8), 571-576. https://doi.org/10.1017/s001216220500112x

Benítez, A., Blanco, A., Aldana, M., Azahares, E. y Almenares, Y. (2012). Actividades físicas recreativas para discapacitados con Parálisis Cerebral Infantil. EFDeportes, 17(170). https://www.efdeportes.com/efd170/actividades-recreativas-paradiscapacitados-con-pc.htm

De Sousa-Peña, L. G., Barra-Danyau, C., Fernández, M., Teixeira, L. G., Casteleti, J. P., Luarte-Rocha, C. y Castelli, L. F. (2020). Limitaciones y Posibilidades en el Entrenamiento del Baloncesto en Silla de Ruedas. Revista Peruana de Ciencias de La Actividad Física y Del Deporte, 7(4), 1036-1044. https://www.rpcafd.com/index.php/rpcafd/article/view/117

Federación Española de Deportes para Ciegos. (2020). Goalball. https://www.fedc.es/deportes/goalball 
Federación Española de Deportes de Personas con Parálisis Cerebral y Daño Cerebral Adquirido. (2020). Federación Española de Deportes de Personas con Parálisis Cerebral y Daño Cerebral Adquirido. http://www.fedpc.org/fedpc-informacioninstitucional.asp

Gamonales, J. M. (2016). La educación física como herramienta de inclusión. Revista Profesional de Investigación, Docencia y Recursos Didácticos, 7o(5), 26-33. https://www.researchgate.net/publication/316455882_La_Educacion_Fisica_co $\underline{\text { mo herramienta de inclusion }}$

Gamonales, J. M. (2017). Fútbol a 5 para personas ciegas como contenido de Educación Física. Revista Profesional de Investigación, Docencia y Recursos Didácticos, 8o(3), 66-70. https://bit.ly/3gLNens

Gamonales, J.M. (2020). Fútbol para personas con discapacidad. En D. Gallado-Vázquez, y S. López-Salas, (eds.), Catálogo de investigación joven en Extremadura (vol. III, pp. 149-152). Servicio de Publicaciones de la Universidad de Extremadura.

Gamonales, J. M., Hidalgo-Murillo, A., León, K., García-Santos, D., \& Muñoz-Jiménez, J. (2018). Propuesta de adaptación del reglamento de balonmano para jugadores con discapacidad intelectual. E-Balonmano.Com: Revista de Ciencias Del Deporte, 14(2), 109-118. https://dialnet.unirioja.es/servlet/articulo?codigo $=6666908$

Gamonales, J. M., León, K., Jiménez, A. y Muñoz-Jiménez, J. (2019). Indicadores de rendimiento deportivo en el fútbol-7 para personas con parálisis cerebral. Revista Internacional de Medicina y Ciencias de la Actividad Física y del Deporte, 19(74). https://doi.org/10.15366/rimcafd2019.74.009

Gamonales, J. M., Muñoz-Jiménez, J., León-Guzmán, K. e Ibáñez, S. J. (2018). 5-a-side football for individuals with visual impairments: A review of the literature.

European Journal of Adapted Physical Activity, 11(1), 4. https://doi.org/10.5507/euj.2018.004

Germán, D. (2001). Deporte y discapacidad. Revista Digital EFDeportes, 7(43). https://www.efdeportes.com/efd43/discap.htm

Gutiérrez-Sanmartín, M. (2004). El valor del deporte en la educación integral del ser humano. Revista de Educación, 335, 105-126.

http://www.revistaeducacion.educacion.es/re335/re335 10.pdf

Revista de Investigación Cuerpo, Cultura y Movimiento ISSN: 2248-4418 | e-ISSN: 2422-474X | DOI: https://doi.org/10.15332/2422474X Vol. 11 N.o 2 | julio-diciembre de 2021 
Hernández-Vázquez, F. J., Labrador-Roca, V., Niort, J., Berbel, G. y Trullols, M. (2016). Respuestas del profesorado de Educación Física ante conflictos con alumnado con discapacidad intelectual y física. Retos, 31, 123-127.

https://doi.org/10.47197/retos.voi31.39861

Lapresa, D., Santesteban, G., Arana, J., Anguera, M. T. y Aragón, S. (2017). Observation System for Analyzing Individual Boccia $\mathrm{BC}_{3}$. Journal of Developmental and Physical Disabilities, 29, 721-734. https://doi.org/10.1007/s10882-017-9552-2

Montilla-Reina, M. J. (2019). Inclusión y competición: Adaptaciones para disminuir las barreras de participación de un grupo de gimnastas con discapacidad intelectual en gimnasia rítmica y propuesta de reglamento específico para la competición. Retos:, 36, 552-56o. https://doi.org/10.47197/retos.v36i36.69283.

Moya, R. M. (2014). Deporte adaptado. CEAPAT-IMS.

Muñoz-Díaz, J.C. (2009). Experiencia de integración en Educación Física: parálisis cerebral. Caleidoscopio, 2. https://multiblog.educacion.navarra.es/jmoreno1/files/2010/07/experiencia_para lisis-cerebral.pdf

Muñoz-Jiménez, J., Gamonales, J. M. y León, K. (2018). El portero de balonmano en silla de ruedas. En M. Antúnez, Innovaciones pedagógicas para el entrenamiento del portero en balonmano (pp. 211-228). Servicio de Publicaciones de la Universidad de Extremadura.

Murphy, N. A. y Carbone, P. S. (2008). Promoting the participation of children with disabilities in sports, recreation and physical activities. Pediatrics, 121(5), 10571061. https://doi.org/10.1542/peds.2008-0566

Pérez-Tejero, J., Reina, R. y Sanz, D. (2012). La Actividad física adaptada para personas con discapacidad en España: perspectivas científicas y de aplicación actual. Cultura, Ciencia y Deporte, 7(21), 213-224. https://doi.org/10.12800/ccd.v7i21.86

Reina, R. (2010). La actividad física y deporte adaptado ante el Espacio Europeo de Enseñanza Superior. Wanceulen.

Reis, R. E., Stdrezk, V. B. y Moreira, P. R. (2020). Footgolf adaptado: o processo de adaptação de uma modalidade para pessoas com deficiência. Revista da Associação Brasileira de Atividade Motora Adaptada, 21(1), 119-134. https://doi.org/10.36311/2674-8681.2020.v21n1.10.p119 
Richards, C. L. y Malouin, F. (2013). Cerebral palsy: definition, assessment and rehabilitation. En O. Dulac, M. Lassonde y H. B. Sarnat (eds.), Handbook Ok Clinical Neurology, (vol. 111, pp. 183-195). https://doi.org/10.1016/B978-0-44452891-9.00018-X

Sanz-Rivas, D. y Reina, R. (2012). Actividades físicas y deportes adaptados para personas con discapacidad. Paidotribo.

Shalock, R. y Verdugo, M. A. (2003). Calidad de vida. Manual para profesionales de la educación, salud y servicios sociales. Alianza.

Shapiro, D., Pitts, B., Hums, M. y Calloway, J. (2012). Infusing disability sport into the sport management curriculum. Choregia: Sport Management International Journal, 8(1), 101-118. https://core.ac.uk/download/pdf/214051962.pdf

Úbeda-Colomer, J., Monforte, J., Campos-Granell, J., Llopis, R., Torregrosa, M. Á. y Devís-Devís, J. (2018). Motivos de práctica y abandono físico-deportivo en alumnado universitario con discapacidad: influencia de la edad y el grado de discapacidad. Cultura, Ciencia y Deporte, 37(14), 51-60.

https://doi.org/10.12800/ccd.v13i37.1038 